\title{
Optimum operation of single cavity photonic switches
}

\author{
Ali Naqavi*a ${ }^{* a}$ Zahra Monem Haghdoost ${ }^{\mathrm{b}}$, Masoud Edalatipour ${ }^{\mathrm{b}}$ \\ Sina Khorasani ${ }^{\mathrm{b}}$ and Khashayar Mehrany ${ }^{\mathrm{b}}$ \\ ${ }^{a}$ Photovoltaics and Thin Film Electronics Laboratory, Institute of Microengineering (IMT) \\ Ecole Polytechnique Fédérale de Lausanne (EPFL), Rue A.- L. Breguet 2 \\ CH-2000 Neuchatel, Switzerland \\ ${ }^{\mathrm{b}} \mathrm{School}$ of Electrical Engineering, Sharif University of Technology, P. O. Box 11365-9363 \\ Tehran, Iran
}

\begin{abstract}
In this work, an optimum frequency is found for the operation of single cavity photonic switches. At this optimum point, the transmission contrast of ON and OFF states takes its highest value, while keeping the device power threshold relatively low and the device speed acceptably high. Then, the dynamic behavior of a typical single cavity all optical switch is investigated in the optimum operation point through temporal Coupled Mode Theory. Switching speed and power are discussed, and the device is shown to be applicable for telecommunication and data processing applications. The analysis is quite general, and can be used for resonant structures, such as photonic crystals and microring resonators, in both side coupled and direct coupled configurations.
\end{abstract}

Keywords: All-optical switching, optical bistability, nonlinear optics, coupled mode theory.

\section{INTRODUCTION}

High speed operation and low power consumption are two main goals to be achieved through all-optical switching ${ }^{1}$; however, both of them cannot be satisfied simultaneously because of the power-bandwidth trade-off. Near the linear resonant frequency of the switch, low power consumption is achieved at the expense of slow operation whereas at offresonance, higher speed is obtained at higher power levels. Furthermore, switching very close to the linear resonance or quite off-resonance results in low contrast between the transmission of ON and OFF states.

Different methods have been used to analyze optical bistable elements which are mainly intended to analyze structures with one-dimensional (1D) periodicity ${ }^{2-4}$; however, the Coupled Mode Theory (CMT) seems to be a good candidate for two-dimensional (2D) and three-dimensional (3D) structure in terms of speed and accuracy. Actually, the CMT has been proved to provide the same result as Finite-Difference Time-Domain (FDTD) for ordinary structures ${ }^{5}$.

The structure of this work is organized as follows. In section 2, Coupled Mode (CM) equations will be written for standing-wave optical switches in two configurations: direct-coupled and side-coupled. At first, the CMT for the linear regime is mentioned. Then, using a perturbation approach ${ }^{6}$, the CMT is extended to the nonlinear case. In section 3 , the optimum switch operation point is found using steady state characteristics obtained in section 2 and in section 4, the transient response of the device is discussed. The device is proved to be able to perform all-optical switching at relatively low power and high speed. Dynamic shift of the resonant frequency of the nonlinear resonator is discussed in section 5 as a complimentary part and it is shown that based on switching dynamics, using characteristics in which the dynamic shift effect is considered will lead to erroneous results.

*ali.naqavi@epfl.ch; phone 032718 3338; pvlab.epfl.ch

Optoelectronic Interconnects and Component Integration IX, edited by Alexei L. Glebov, Ray T. Chen, Proc. of SPIE Vol. 7607, 760719 - (C) 2010 SPIE · CCC code: 0277-786X/10/\$18 · doi: 10.1117/12.840463 


\section{COUPLED MODE THEORY FOR A STANDING WAVE SINGLE RESONATOR OPTICAL SWICTCH}

Two major configurations to realize optical bistability, the direct-coupled and the side-coupled geometries, are shown in figure 1. In the case of direct-coupled geometry, transmission spectrum has a peak at resonance while for the sidecoupled, it has a dip. In this way, the two structures are the duals of each other.
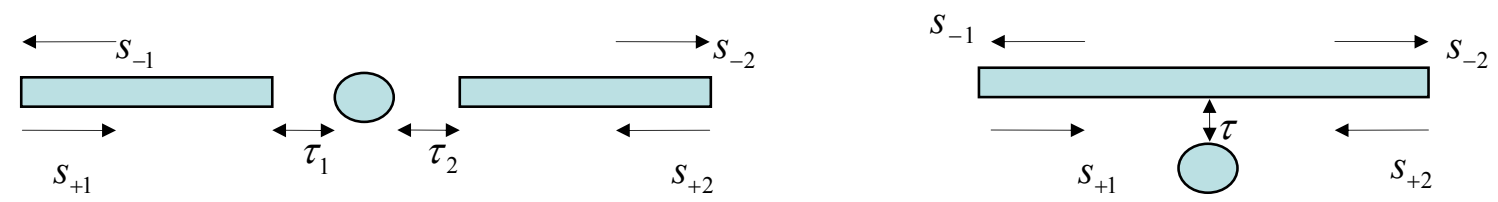

Figure 1. Two major configurations for single resonator optical switches: direct-coupled (left) and side-coupled (right).

In the direct coupled geometry, one can write the $\mathrm{CM}$ equation for the wave in the resonator as ${ }^{7}$

$$
\frac{d a}{d t}=\left(j \omega_{0}-\frac{1}{\tau_{0}}-\frac{1}{\tau_{1}}-\frac{1}{\tau_{2}}\right) a+\kappa_{1} s_{+1}+\kappa_{2} s_{+2},
$$

where $a$ denotes the wave in resonator ${ }^{7}, \tau_{0}$ is associated with the resonator internal loss, and $\tau_{i}$ refers to the coupling between the resonator and waveguide $i$ due to the leakage of resonator energy to the waveguide. The resonator resonant frequency at the absence of waveguides is $\omega_{0} . S_{+i}$ represents the input wave of the $i$-the waveguide, $S_{-i}$ is the output wave of the $i$-th waveguide and $\kappa_{i}$ stands for the coupling coefficient between the resonator and the input wave of waveguide $i$. These parameters are given by

$$
\kappa_{i}=\sqrt{\frac{2}{\tau_{i}}}, i=1,2
$$

and

$$
\begin{gathered}
s_{+1}(t)=s_{-1}(t)+\kappa_{1}^{*} a(t), \\
s_{+2}(t)=s_{-2}(t)+\kappa_{2}^{*} a(t) .
\end{gathered}
$$

Similarly, for the side-coupled configuration the following relation can be concluded

$$
\frac{d a}{d t}=\left(j \omega_{0}-\frac{1}{\tau_{0}}-\frac{1}{\tau}\right) a+\kappa\left(s_{+1}+s_{+2}\right),
$$

provided that

$$
\kappa=\sqrt{\frac{1}{\tau}}
$$

and

$$
\begin{aligned}
& s_{+1}(t)=s_{-2}(t)+\kappa^{*} a(t), \\
& s_{+2}(t)=s_{-1}(t)+\kappa^{*} a(t) .
\end{aligned}
$$


The effect of nonlinearity is added to the formulation simply by changing the resonant frequency ${ }^{6}$, i.e.

$$
\begin{gathered}
\omega_{0} \rightarrow \omega_{0}(1-\xi), \\
\xi=\frac{P}{2 P_{0} Q},
\end{gathered}
$$

In the latter relation, $Q$ is the cavity quality factor, $P_{0}$ is the characteristic power of the device ${ }^{5}$ and $P$ is the power coupled from the cavity to the waveguide(s). If monochromatic input is considered only from one side i.e. $s_{+1}=\cos (\omega t)$ and $s_{+2}=0$, as is normally encountered, steady state power transmission for the direct-coupled geometry can be found as ${ }^{8}$

$$
T=\frac{P_{\text {out }}}{P_{\text {in }}}=\frac{1}{1+\left(P_{\text {out }} / P_{0}-d\right)^{2}} .
$$

and if in the right hand side of equation (8) we substitute $P_{\text {out }}$ for $P_{\text {ref }}$, the steady state power reflection for the sidecoupled structure can be obtained as ${ }^{5}$

$$
R=\frac{P_{r e f}}{P_{i n}}=\frac{1}{1+\left(P_{r e f} / P_{0}-d\right)^{2}} .
$$

In the two latter relations $\delta$ represents the normalized frequency detuning ${ }^{5}, P_{\text {out }}=\left|S_{-2}\right|^{2}$ and $P_{r e f}=\left|S_{-1}\right|^{2}$. Similarly, we have $P_{i n}=\left|s_{+1}\right|^{2}$.

\section{FINDING THE OPTIMUM OPERATION FREQUENCY}

For the sake of clarity, we define the meaning of optimum operation in this work. The optimum operation frequency is a frequency at which the two states of the output power are as distinct as possible. The best figure of merit we found to evaluate this property is the value of jump in transmission/ reflection characteristic of the device. Based on (8) and (9), bistable transmission is exhibited when $\delta>\sqrt{3}$. Figure 2 shows the power characteristic and the transmission of the direct-coupled geometry at $\delta=3.5$ and bistability is clearly observed. Our aim is to maximize the distinction between the two states. For this purpose, however, the OFF to ON jump, $\Delta$, is a bottleneck; therefore, getting the maximum value of $\Delta$ in figure 2 (left) is of interest. Note that in both direct- and side-coupled geometries, no bistability is obtained for $\delta<\sqrt{3}$ and the jump value will be quite small for very large $\delta$. Manipulating some mathematical calculations, one can find the OFF to ON jump value in the power characteristic - Figure 2 (right)

$$
\Delta_{p}=\frac{27}{2\left[-3+\delta^{2}+\frac{\delta\left(9+\delta^{2}\right)}{\sqrt{\delta^{2}-3}}\right]} .
$$

A simple differentiation with respect to $\delta$ and finding the roots reveals that the optimum value of the normalized detuning is

$$
\delta_{\text {opt }}=2.32 \text {. }
$$



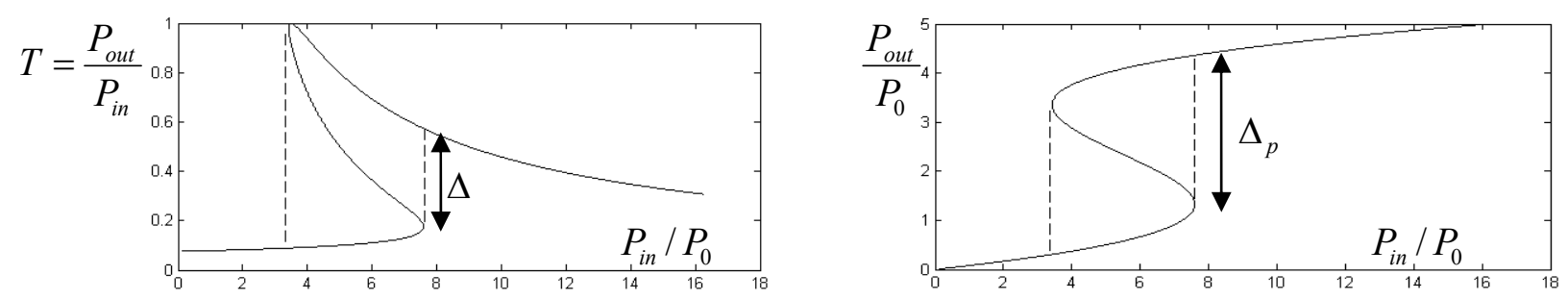

Figure 2. Transmission (left) and power characteristic (right) of the direct-coupled structure at $\delta=3.5$.

\section{DISCUSSION ON THE TIME DOMAIN RESPONSE}

The optimum normalized frequency detuning in (11) was found with frequency domain characteristics. However, the time domain performance of the switch should be investigated, to verify whether the switch can operate effectively at $\delta_{\text {opt }}=2.32$ or not. At frequencies very close to the linear resonant frequency, $\omega_{0}$, ultrafast switching is quite difficult due to transient effects. In this section we verify that the result obtained in (11) allows switching at very high speeds.

From either (1) or (4), one can simply develop a finite difference scheme to find the time evolution of the wave in the resonator $a$, and thus the other parameters, e.g. the output power $\left|S_{-2}\right|^{2}$, could be obtained easily. In the case of a symmetric direct-coupled structure, equation (4) holds for both configurations with the assumption $\tau^{-1}=\tau_{1}^{-1}+\tau_{2}^{-1}$ for the direct coupled case. Furthermore, from relations (3-b) and (6-b) and the fact that $s_{+2}=0$ one can easily find out that $P_{\text {out }}$ of the direct-coupled structure and $P_{r e f}$ of the side-coupled geometry show the same behavior not only in the steady state regime, but also in the transients.

The output of both configurations was found through a CM simulation with parameters $Q=557, \quad P_{0}=77 \mathrm{~mW}$, $\lambda_{0}=2 \pi c / \omega_{0}=1.55 \mu \mathrm{m}$, and $\delta=2.3$. Time step duration was set $\Delta t_{0}=0.005 \pi / \omega_{0}$ and the simulation lasted for $2 \times 10^{6}$ time steps. The input power envelope was of the form $A_{0} \exp \left[-\alpha\left(t-t_{m}\right)^{2} / t_{m}{ }^{2}\right]$ where $A_{0}=5 P_{0}, \alpha=5$ and $t_{m}$ stands for the middle time of the simulation.
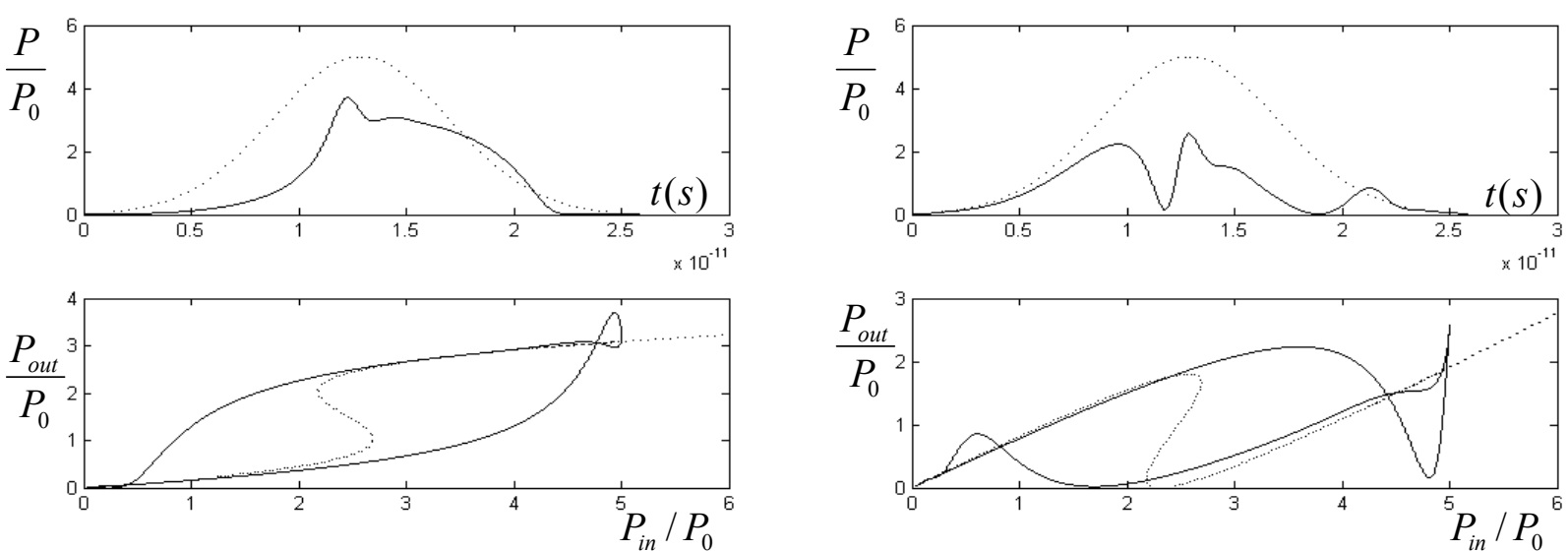

Figure 3. Dynamics of the direct-coupled (left) and side-coupled (right) structures. Up: Time evolution of output power (solid) and input power (dotted). Bottom: Transient (solid) and steady-state (dotted) power characteristics. The transient power characteristic is obtained by plotting output wave versus input power and the steady-state relation is found using relations (8) and (9). 
Through simulation results shown in figure 3, one can find out that optical switching has been maintained successfully for the input pulses of about $10 \mathrm{ps}$ duration in both configurations. The input power is relatively low since the input carrier frequency is not very far from the cavity resonant frequency in the linear regime. Furthermore, the transmission in the ON state is acceptably high and the two states can be distinguished very well. The deviation of the time domain curves from the third order steady state curve is a result of the very fast switching. As the input pulse is widened, the transient characteristic approaches the steady state result ${ }^{1}$ except for the unstable part which cannot be observed physically. Figure 4 verifies the latter claim again; in very short pulse durations, the output does not show bistable behavior since in such cases there is not enough time for the light and matter to interact effectively.
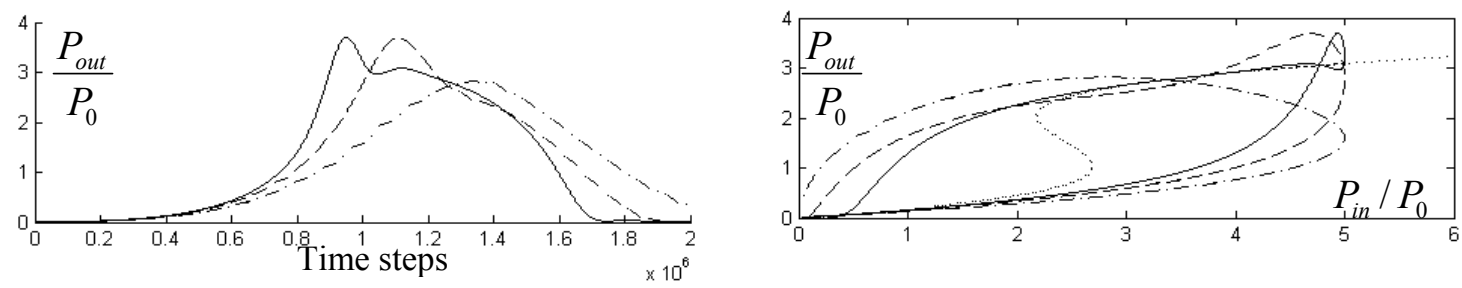

Figure 4. Output power waveform for different values of time step duration (left) and transient power characteristic (right) for $\Delta t_{0}$ (solid), $\Delta t_{0} / 2$ (dashed), $\Delta t_{0} / 5$ (dotted-dashed). The dotted curve is the third order steady-state characteristic. The figures are plotted for the direct-coupled configuration. For the side-coupled geometry similar results can be found.

\section{THE DYNAMIC SHIFT EFFECT}

In this part of the paper, we compare the output power characteristics obtained through the CMT with the power characteristics found in a recent work ${ }^{9}$ in which, the effect of the pulsating behavior of the resonator field is considered to get more realistic results. One may assume the standing wave in the resonator varies proportional to $\cos (\omega t)$. Hence, the nonlinear material in the resonator will exhibit changes in the index proportional to $\cos ^{2}(\omega t)$ and the resonant frequency will not be fixed due to its dependence on the index of the nonlinear material. This effect has been referred to as the Dynamic Shift Effect (DSE) ${ }^{9}$. As in the process of obtaining (8) and (9) such dependence has not been accounted for, it is claimed that the steady state characteristic described through relations (8) and (9) is only an optimistic approximation and should be modified ${ }^{9}$.

In figure 5, the power characteristic found through relation (1) is plotted together with the steady state power characteristic in which DSE modification is considered ${ }^{9}$ and the steady state power characteristic of (8). Clearly, the transient curve is much closer to the curve associated to (8) than the other curve. Therefore, considering the dynamic frequency shift of the resonant frequency has not lead to a better result. To justify the difference between the transient response and the one DSE result, one should notice that by taking the DSE into account, the effective value of the output power is calculated ${ }^{9}$ which is not useful while considering transient effects.

\section{CONCLUSION}

Low power and high speed have been two goals of optical switching between which one should make a compromise. In this paper an optimum frequency is found for the operation of standing wave single cavity photonic switches. The power-speed trade-off has been taken into account at the same time with providing the highest possible distinction between the two states. Transient analysis has been performed with the help of CMT to verify the possibility of ultrafast switching. Also, it is shown that the dynamic shift effect should not be considered when maintaining the transient response of the device. 


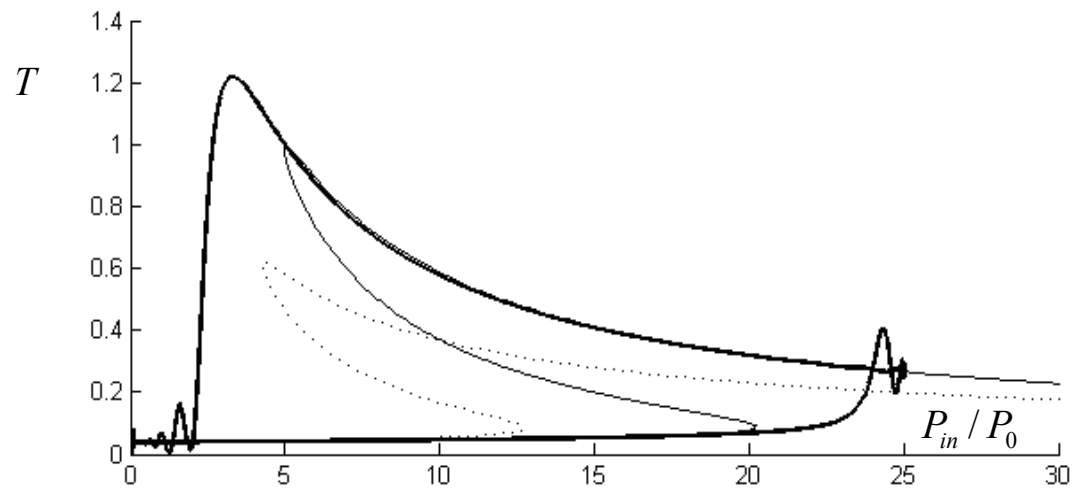

Figure 5. Power characteristic obtained from temporal CMT (thick solid), steady-state CMT (thin solid) and steady-state CMT considering DSE (dotted). Simulation parameters are taken $Q=50, P_{0}=9.5557 \mathrm{~W}, \lambda_{0}=1.55 \mu \mathrm{m}$ and $\delta=5$.

\section{REFERENCES}

[1] Naqavi, A., Abediasl, H., Mehrany, K., Khorasani, S., Chamanzar, M. and Adibi, A., "On the power-bandwidth trade-off in bistable photonic switches," Proc. SPIE 7223, 722308 (2009).

[2] Maywar, D., N. and Agrawal, G., P., "Transfer-matrix analysis of optical bistability in DFB semiconductor laser amplifiers with nonuniform gratings," IEEE J. Quantum Electron. 33(1), 2029-2037 (1997).

[3] Zamani Aghaie, K., Rabbani, M. and Shahabadi, M., "Multiple-scale analysis of optical bistability in semiconductor periodic structures," J. of Opt. Soc. Am. 24(4), 887-894 (2007).

[4] Janyani, A., Paul, V., Vukovic, P. Benson, T., M. and Sewell, P., "TLM modeling of nonlinear optical effects in fibre bragg gratings," IEE Proc. Optoelectron. 151(4), 185-192 (2004).

[5] Yanik, M. F., Fan, S. and Soliačić, M., "High-contrast all-optical bistable switching in photonic crystal microcavities," Appl. Phys. Lett., 83(14), 2739-2741 (2003).

[6] Bravo-Abad, J., Fan, S., Johnson, S., G., Joannopoulos, J., D. and Soliačić, M., "Modeling nonlinear optical phenomena in nanophotonics," J. Lightwave Technol., 25(9), 2539-2546 (2007).

[7] Haus, H., A., [Waves and Fields in Optoelectronics], Prentice Hall, New Jersey, 197-234 (1984).

[8] Soliačić, M., Ibanescu, M., Johnson, S., G., Fink, Y. and Joannopoulos, J., D., "Optimal bistable switching in nonlinear photonic crystals," Phys. Rev. E 66(055601(R)), 1-4 (2002).

[9] Lin, X., S., Shi, Q., Cai, X., H. and Zhao, N., S., "Dynamic shift effect on bistability in nonlinear optical microcavities," Opt. Express, 14(15), 6794-6799 (2006). 